\title{
Pacific
}

Journal of

Mathematics

\section{STABILITY OF NONSINGULAR GROUP ORBITS}

CLARK DEAN Horton 


\title{
STABILITY OF NON-SINGULAR GROUP ORBITS
}

\author{
Clark D. Horton
}

\begin{abstract}
Let $G$ be a compact Lie group of isometries acting on a riemannian manifold $M$. In recent years, there has been a great deal of interest in minimal submanifolds that arise as orbits of such an action. In this paper, we formulate necessary and sufficient conditions for the stability of minimal codimension two principal orbits. These conditions are expressed in terms of the eigenvalues of a $G$-invariant vector field on the orbit, the eigenvalues of the laplacian of the orbit, and the eigenvalues of the hessian of the volume function. Next we use a poincare inequality along with the orthogonality relations on the group $G$ to find conditions for the stability of exceptional orbits. These conditions are used to find new examples of stable minimal submanifolds in the generalized lens-spaces and the quaternionic space forms.
\end{abstract}

1. Introduction. Let $M$ be a riemannian manifold with a compact Lie group of isometries $G$ acting on the left. W. Y. Hsiang and H. B. Lawson [HL] have discovered a very simple condition for an orbit of $G$ to be a minimal submanifold. They showed that an orbit is minimal if its first variation is zero under equivariant deformations. It then follows that a principal orbit $P$ is minimal if the volume function $\tilde{\nu}: M \rightarrow R$ is critical on $P$, where $\tilde{\nu}(p)$ is defined to be the volume of the orbit through $p$. Also, an orbit is minimal if all of the orbits in a surrounding tubular neighborhood are of a higher type. A natural question to ask is when are these minimal orbits stable or unstable.

In this direction, $\mathbf{J}$. Brothers [Br1] has produced a second variation formula that is applicable to the case of a minimal principal orbit. From this formula, it follows that a necessary condition for the stability of a principal orbit is that $\tilde{\nu}$ have positive semi-definite hessian. His sufficient conditions for stability involve bounds on the lengths of certain $G$-invariant vector fields on the orbit. However, if the normal distribution to the orbit is involutive, then a positive definite hessian is sufficient for stability. Although these conditions have led to many new examples of stable principal orbits, they are not sharp in general. Through the use of Brothers' second variation formula, we show that the Jacobi operator can be decomposed into a sum of two commuting self-adjoint operators. This is used to find precise conditions for 
the stability of dimension one and codimension two minimal principal orbits. These conditions are expressed in terms of the eigenvalues of the hessian matrix of the volume function, the eigenvalues of the laplacian on the orbit, and the length and eigenvalues of a $G$-invariant vector field. Precise conditions are then given for the stability of equivariant minimal embeddings of codimension two spheres. In order to treat the situation of minimal exceptional orbits, the canonical form of a tubular neighborhood is studied. A variation of the exceptional orbit is then lifted to the principal orbit where Brothers' second variation formula is applied. Special properties of the lift along with some elementary facts from group representation theory are used to find conditions for stability. These results are then used to find new examples of stable minimal submanifolds in the generalized Lens-spaces and in the quaternionic space forms. It also follows that, $R P^{n}$ is stable in $R P^{m}$ for $m<n$.

Finally, I would like to thank John Brothers for his many helpful discussions and suggestions during the preparation of this work.

2. Preliminaries. Throughout this paper $G$ will denote a compact connected Lie group of isometries of some riemannian manifold unless otherwise stated. The action of $G$ is always assumed to be effective. If $x$ is a point in the manifold on which $G$ acts, then the orbit through $x$ is denoted by $G(x)$ and the isotropy group is denoted by $G_{x}$. We may put an equivalence relation on the set of group orbits by declaring two orbits to be equivalent if their isotropy groups are conjugate. An equivalence class of orbits is called an orbit type. If $G_{x}$ and $G_{y}$ are isotropy groups with $\left(G_{x}\right)$ and $\left(G_{y}\right)$ denoting their equivalence classes, we may put a partial ordering on them by saying that $\left(G_{x}\right) \geq\left(G_{y}\right)$ if and only if $G_{x}$ is conjugate to a subgroup of $G_{y}$. There exists a unique orbit type $\left(G_{x_{0}}\right)$, called the principal orbit type with the property that $\left(G_{x_{0}}\right) \geq\left(G_{x}\right)$ for all $x \in M$. An orbit that belongs to the principal orbit type is said to be a principal orbit. The union of the set of principal orbits is an open dense subset of the manifold on which $G$ acts [B]. An orbit with isotropy group $G_{x}$ is said to be exceptional if some conjugate of the isotropy group of a principal orbit has finite index in $G_{x}$.

Let $H$ be a closed subgroup of $G$ with a linear action on a vector space $V$. We may define an action of $H$ on $G \times V$ by $h(g, v)=$ $\left(g h^{-1}, h v\right)$. The orbit of the point $(g, v)$ is denoted by $[g, v]$. The orbit space is denoted by $G \times{ }_{H} V$ and is called a fibered product. Let $G$ act on a riemannian manifold $M$, and let $x \in M$ with isotropy 
group $G_{x}$. Then the orbit of $G$ through $x$ has a tubular neighborhood equivariantly diffeomorphic to the fibered product $G \times_{G_{x}} V$ where $V$ is the space of vectors normal to the orbit at $x$ and the action of $G_{x}$ is the action on $T_{x} M[\mathbf{B}]$. If the orbit is principal, then the action of $G_{x}$ on the normal space is trivial so that a principal orbit $P$ has a trivial normal bundle [B]. A group orbit is said to be isolated if it has a tubular neighborhood in which every other orbit is of a higher type.

Let $M$ be a riemannian manifold with compact submanifold $N$ of dimension $n$ without boundary. Let $\mathscr{H}^{n}$ denote the Hausdorff $n$-measure on $M$ induced by the riemannian metric and let $W$ be a smooth vector field in a neighborhood of $N$. Denote the flow of $W$ by $\phi_{t}$. For $k=1,2$ the $k$ th variation of the area of $N$ with respect to the deformation vector field $W$ is defined by

$$
\delta^{(k)}(W)=\left.\frac{d^{k}}{d t^{k}} \mathscr{H}^{n}\left(\phi_{t} N\right)\right|_{t=0}
$$

We say that $N$ is minimal if $\delta^{(1)}(W)=0$ for all vector fields $W$ and stable if $\delta^{(2)}(W) \geq 0$ for all vector fields $W$.

If $f \in C^{\infty}(M)$, has a critical point at $x \in M$, then one defines the hessian $H$ of $f$ at $x$ to be the symmetric bilinear function on $T_{x} M$ such that $H(v, w)=V \circ W(f)(x)$ where $V$ and $W$ are smooth extensions of $v, w \in T_{x} M$ to a neighborhood of $x$. Suppose we have a group action on a riemannian manifold $M$ whose orbits are principal with dimension $d$. We define a real valued function $\tilde{\nu}$ on $M$ where $\tilde{\nu}(p) \equiv \mathscr{H}^{d}(P)$ where $P$ is the principal orbit through $p \in M . P$ is minimal if and only if $\tilde{\nu}$ is critical at $p \in P$ [HL]. If $\lambda_{1}$ is the first eigenvalue of the laplacian $\Delta$ on $M$ and $f \in C^{\infty}(M)$ with $\int_{M} f=0$, then we have the Poincaré inequality

$$
\lambda_{1} \int_{M}\|d f\|^{2} \geq \int_{M} f^{2}
$$

3. Principal orbits. Let $P$ be a principal orbit arising from the action of a group $G$. We may choose a normal, $G$-invariant, orthonormal, frame field $W_{1}, \ldots, W_{n}$ on $P$ where $n$ is the codimension of $P$ in the manifold on which $G$ acts. Every normal vector field $W$ on $P$ can be written $W=\sum_{i=1}^{n} A^{i} W_{i}$ where $A^{i}: P \rightarrow R$ for each $i$. If we take the projections of the vectors $\left[W_{i}, W_{k}\right]$ on $P$ we get $G$ invariant vector fields $W_{i k}$ for $i, k=1, \ldots, n$ on $P$. If the $W_{i k}=0$ for $i, k=1, \ldots, n$ then we say that the normal distribution to $P$ is involutive. (See [Br1].) 
Brothers [Br1] has derived the following formula for the second variation of minimal $P$ :

$$
\delta_{P}^{(2)}(W)=\int_{P} \tilde{\nu}^{-1} W^{2} \tilde{\nu}+\sum_{i=1}^{n}\left\|d A^{i}\right\|^{2}-\sum_{i<j}\left\langle W_{i k}, A^{i} d A^{k}-A^{k} d A^{i}\right\rangle .
$$

We observe that Brothers' formula can be rewritten in a way that is more illuminating. Notice that

$$
\int_{P}\left\|d A^{i}\right\|^{2}=\int_{P}\left\langle d A^{i}, d A^{i}\right\rangle=\int_{P} A^{i} \Delta A^{i}
$$

so that we can write

$$
\int_{P} \sum_{i=1}^{n}\left\|d A^{i}\right\|^{2}=\int_{P} \vec{A}^{t} \widetilde{\Delta} \vec{A}
$$

where

$$
\vec{A} \equiv\left(\begin{array}{c}
A^{1} \\
\vdots \\
A^{n}
\end{array}\right)
$$

and

$$
\widetilde{\Delta} \equiv\left(\begin{array}{cccc}
\Delta & & & \\
& \Delta & 0 & \\
& 0 & \ddots & \\
& & & \Delta
\end{array}\right) .
$$

The third term in Brothers' formula can be written as

$$
\int_{P} \vec{A}^{t} S \vec{A}
$$

where

$$
S \equiv\left(\begin{array}{ccccc}
0 & -W_{12} & -W_{13} & \ldots & -W_{1 n} \\
W_{12} & 0 & -W_{23} & \ldots & -W_{2 n} \\
W_{13} & W_{23} & 0 & \ldots & -W_{3 n} \\
\vdots & \vdots & \vdots & \ddots & \vdots \\
W_{1 n} & W_{2 n} & W_{3 n} & \ldots & 0
\end{array}\right)
$$

Finally, since $\tilde{\nu}$ has a critical point on the orbit $P$, we can thus write the first term as

$$
\int_{P} \vec{A}^{t} H \vec{A}
$$

where $H$ is the $n$ by $n$ matrix with constant entries $H_{i j}=\tilde{\nu}^{-1} W_{i} W_{j} \tilde{\nu}$. Thus

$$
\delta_{P}^{(2)}(W)=\int_{P} \vec{A}^{t} J \vec{A}
$$


where $J \equiv S+H+\widetilde{\Delta}$ is the well-known Jacobi operator. The matrices $J, S, H$, and $\widetilde{\Delta}$ may be regarded as linear operators on the space $\bigoplus_{i=1}^{n} C^{\infty}(P)$. Clearly $H \widetilde{\Delta}=\widetilde{\Delta} H$ since $H$ is constant and $\widetilde{\Delta}$ is diagonal with equal entries.

LEMMA 3.1. Let $M$ be a compact riemannian manifold without boundary and $X$ a smooth vector field on $M$ such that $\operatorname{div} X=0$. Then $X$ is a skew-symmetric operator on functions.

Proof. Assume that $M$ is orientable with volume form $\omega$. Let $f, g \in C^{\infty}(M)$. If $L_{X}$ denotes the Lie derivative by $X$ we then have

$$
L_{X}(f g \omega)=(f X g+g X f) \omega+(f g \operatorname{div} X) \omega
$$

which follows by Leibniz's rule and the identity $L_{X} \omega=(\operatorname{div} X) \omega$ [P]. We then get

$$
\int_{M}(f X g+g X f) \omega=\int_{M} L_{X}(f g \omega) .
$$

We now use the identity $L_{X} \omega=\left(d \circ l_{X}+l_{X} \circ d\right) \omega$, where $l_{X} \omega$ is the $(n-1)$-form defined by $\left(l_{X} \omega\right)\left(v_{1}, \ldots, v_{n-1}\right) \equiv \omega\left(X, v_{1}, \ldots, v_{n-1}\right)$ [W], and Stokes' theorem to write the last integral as

$$
\int_{M}\left(d \circ l_{X}+l_{X} \circ d\right)(f g \omega)=\int_{M} d \circ l_{X}(f g \omega)=0 .
$$

Hence $\int_{M} f X g=-\int_{M} g X f$. If $M$ is not orientable then we pass to an oriented double cover.

LEMMA 3.2. Let $G$ be a compact Lie group equipped with a biinvariant metric and let $H$ be a closed subgroup of $G$. Furthermore, suppose that $G / H$ has a metric such that the canonical projection $\pi: G \rightarrow G / H$ is a riemannian submersion. Then any $G$-invariant vector field $X$ on $G / H$ is Killing.

Proof. Because $\pi: G \rightarrow G / H$ is a submersion, we may lift $X$ to a left-invariant vector field $\widetilde{X}$ on $G$ which is normal to the fibers of $\pi$. Since the metric on $G$ is bi-invariant, the flow $\tilde{\phi}_{t}$ of $\widetilde{X}$ is an isometry for each $t$. Since the submersion is riemannian, it follows that the flow $\phi_{t}$ of $X$ is an isometry for each $t$.

Proposition 3.3. Let $P$ be a principal orbit with a metric for which the vector fields $W_{i k}, i, k=1, \ldots, n$ are Killing, and let $J, H, S$, 
and $\widetilde{\Delta}$ be as above. Then
(a) $\widetilde{\Delta} S=S \widetilde{\Delta}$,
(b) $\widetilde{\Delta}^{*}=\tilde{\Delta}$,
(c) $S^{*}=S$,
(d) $\widetilde{\Delta}(S+H)=(S+H) \widetilde{\Delta}$,
(e) $H^{*}=H$.

Proof. (a) follows from the fact that the laplacian commutes with Killing vector fields. For (b) and (c) we make use of the fact that the adjoint of a matrix of operators is obtained by replacing each entry by its adjoint and then transposing, along with the facts that $\Delta$ is a self-adjoint operator on $C^{\infty}(P)$ and each $W_{i k}$ is a skew-symmetric operator on $C^{\infty}(P)$ (Lemma 3.1). (d) is implied by (a) and the remark preceding Lemma 3.1. Finally (e) is true since $H$ is a constant times the restriction to the normal bundle of $P$ of the hessian matrix of the function $\tilde{\nu}$.

The proposition implies that the operator $J$ is the sum of two commuting self-adjoint operators $\widetilde{\Delta}$ and $S+H$. The eigenvalues of $\widetilde{\Delta}$ are clearly the same as the eigenvalues of $\Delta$. Since the eigenspaces of $\widetilde{\Delta}$ are finite dimensional and $S+H$ commutes with $\widetilde{\Delta}$, we see that there exists a basis of $\bigoplus_{i=1}^{n} C^{\infty}(P)$ for which both operators are diagonal. Let $\phi_{1}, \phi_{2}, \ldots$ be a basis for the set of common eigenvectors for $\widetilde{\Delta}$ and $H+S$, and suppose that $\widetilde{\Delta} \phi_{k}=\lambda_{k} \phi_{k}$ and $(H+S) \phi_{k}=\rho_{k} \phi_{k}$, where $0<\lambda_{0}<\lambda_{1}<\cdots$. Then the eigenvalues for $J$ are $\left\{\lambda_{k}+\rho_{k}: k=0, \ldots\right\}$. Thus we see that in order for $\delta_{P}^{(2)}(W)=\int_{P} \vec{A}^{t} J \vec{A}$ to be non-negative for all $W$, it must be true that $J$ has non-negative eigenvalues. Therefore we have

TheOREM 3.4. Let $P$ be a minimal principal orbit for which the $W_{i k}, i, k=1, \ldots, n$ are Killing. Then $P$ is stable if and only if $\lambda_{k}+\rho_{k} \geq 0$ for all $k$.

REMARKs. The requirement that the $W_{i k}$ be Killing vector fields seems to be a bit restrictive. However, Lemma 3.2 gives a general condition for which a $G$-invariant vector field on an orbit is Killing. The standard metrics on the rank one symmetric spaces satisfy this condition, so that the requirement is not a problem in most of the interesting situations. Also because the hessian is symmetric, we may assume that it is in diagonal form with eigenvalues $\mu_{1}, \ldots, \mu_{n}$.

Suppose $P$ is a one-dimensional orbit. Such an orbit is diffeomorphic to $S^{1}$. Hence the laplacian is $-d^{2} / d \theta^{2}$. Let $L$ be the length of 
the orbit. Then the $k$ th eigenspace of $\Delta$ is

$$
I_{k}=\{a \sin (2 \pi k / L)+b \cos (2 \pi k / L): a, b \in R\}
$$

and the $k$ th eigenvalue is $(2 \pi k / L)^{2}, k=0,1, \ldots$ Each $W_{i j}$ may be written as $A_{i j} d / d \theta$ where $A_{i j}$ is a scalar and $W_{i j}$ is clearly Killing for all $i$ and $j$. Applying Theorem 3.4 we have

Corollary 3.5. Let $P$ be a one-dimensional minimal principal orbit. Then $P$ is stable if

$$
\inf _{k}\left(\frac{4 \pi^{2} k^{2}}{L^{2}}+R_{k}\right) \geq 0
$$

where $R_{k}$ is the smallest eigenvalue of the $2 n \times 2 n$ matrix $\left(K_{p q}\right)$, $p, q=1, \ldots, n$, with

$$
K_{p p}=\left(\begin{array}{cc}
\mu_{p} & 0 \\
0 & \mu_{p}
\end{array}\right), \quad K_{p q}=\left(\begin{array}{cc}
0 & -\frac{2 \pi k}{L} A_{p q} \\
\frac{2 \pi k}{L} A_{p q} & 0
\end{array}\right), \quad p \neq q .
$$

EXAMPLE 3.6. Consider the Lie group $U(2)$ whose Lie algebra is spanned by the matrices

$$
\begin{aligned}
D_{1}=\left(\begin{array}{ll}
i & 0 \\
0 & 0
\end{array}\right), \quad D_{2} & =\left(\begin{array}{ll}
0 & 0 \\
0 & i
\end{array}\right), \quad I=\frac{1}{\sqrt{2}}\left(\begin{array}{cc}
0 & -1 \\
1 & 0
\end{array}\right), \\
J & =\frac{1}{\sqrt{2}}\left(\begin{array}{ll}
0 & i \\
i & 0
\end{array}\right)
\end{aligned}
$$

which form an orthonormal basis with respect to the inner product given by $\langle A, B\rangle \equiv \operatorname{tr}\left(A \bar{B}^{t}\right)$. Extend this to a bi-invariant metric on $U(2)$. Define a left action of $S^{1}$ on $U(2)$ by $\rho: S^{1} \times U(2) \rightarrow U(2)$ with

$$
\rho\left(e^{i \theta}, A\right)=\left(\begin{array}{cc}
e^{i \theta} z_{11} & e^{i \theta} z_{12} \\
z_{21} & z_{22}
\end{array}\right)
$$

where $A \in U(2)$. Each of the orbits is a right coset of the one parameter subgroup given by $t \rightarrow \exp t D_{1}$. Each orbit is minimal with constant length $2 \pi$ hence the hessian of the length function of the orbit zero. The frame $W_{1}, W_{2}, W_{3}$ for each of the orbits is given by the restriction of the left-invariant vector fields represented by $D_{2}$, $I$, and $J$ respectively. A simple computation gives $W_{12}=W_{13}=0$, and $W_{23}=-D_{1}$. In this case $R_{k}=-k$. Hence, by Corollary 3.5 we have stability if $\left(k^{2}-k\right) \geq 0$ for all $k$. This is clearly the case, so we conclude that the orbits are stable geodesics. 
If $P$ is a principal orbit of codimension two, then the operator $J$ can be written

$$
J=\left(\begin{array}{cc}
\Delta & 0 \\
0 & \Delta
\end{array}\right)+\left(\begin{array}{cc}
\mu_{1} & -W_{12} \\
W_{12} & \mu_{2}
\end{array}\right)
$$

Now assume that the $G$-invariant vector field $W_{12}$ is Killing. We write $W_{12}=\left\|W_{12}\right\| \widehat{W}_{12}$ where $\widehat{W}_{12}$ is a unit vector field on $P$ and $\left\|W_{12}\right\|$ is constant. Let $0<\lambda_{1}<\lambda_{2}<\cdots$ be the eigenvalues of $\Delta$ on $P$ and write $C^{\infty}(P) \oplus C^{\infty}(P)=\bigoplus_{k=1}^{\infty} E_{k}$ where

$$
E_{k}=\left\{\left(\begin{array}{c}
\phi_{1} \\
\phi_{2}
\end{array}\right): \Delta \phi_{i}=\lambda_{k} \phi_{i}, i=1,2\right\}
$$

with $\operatorname{dim} E_{k}<\infty$. Since $\Delta \widehat{W}_{12}=\widehat{W}_{12} \Delta$ we see that $\widehat{W}_{12}$ has a finite number of eigenvalues on $E_{k}$. Let $\sigma_{k}$ be the largest eigenvalue of $-\widehat{W}_{12}^{2}$ on $E_{k}$.

THEOREM 3.7. Let $P$ be a minimal, codimension two, principal orbit for which $W_{12}$ is a Killing vector field. Then $P$ is stable if and only if

$$
\left\|W_{12}\right\|^{2} \leq \inf _{k}\left(\frac{\left(\lambda_{k}+\mu_{1}\right)\left(\lambda_{k}+\mu_{2}\right)}{\sigma_{k}}: \sigma_{k}>0\right) \text {. }
$$

Proof. Consider the eigenvalue problem

$$
\left(\begin{array}{cc}
\mu_{1} & -\left\|W_{12}\right\| \widehat{W}_{12} \\
\left\|W_{12}\right\| \widehat{W}_{12} & \mu_{2}
\end{array}\right)\left(\begin{array}{c}
\phi_{1} \\
\phi_{2}
\end{array}\right)=\alpha\left(\begin{array}{c}
\phi_{1} \\
\phi_{2}
\end{array}\right), \quad\left(\begin{array}{c}
\phi_{1} \\
\phi_{2}
\end{array}\right) \in E_{k} .
$$

We have

$$
-\widehat{W}_{12} \phi_{2}=\left(\frac{\alpha-\mu_{1}}{\left\|W_{12}\right\|}\right) \phi_{1}
$$

and

$$
\widehat{W}_{12} \phi_{1}=\left(\frac{\alpha-\mu_{2}}{\left\|W_{12}\right\|}\right) \phi_{2}
$$

Applying $\widehat{W}_{12}$ to both sides and combining we get

$$
-\widehat{W}_{12}^{2} \phi_{2}=\frac{\left(\alpha-\mu_{1}\right)\left(\alpha-\mu_{2}\right)}{\left\|W_{12}\right\|^{2}} \phi_{2}
$$

and

$$
-\widehat{W}_{12}^{2} \phi_{1}=\frac{\left(\alpha-\mu_{1}\right)\left(\alpha-\mu_{2}\right)}{\left\|W_{12}\right\|^{2}} \phi_{1} .
$$

Hence we see that $\left(\alpha-\mu_{1}\right)\left(\alpha-\mu_{2}\right) /\left\|W_{12}\right\|^{2}$ is an eigenvalue of $-\widehat{W}_{12}^{2}$ on $E_{k}$. If we let $0 \leq \varepsilon_{1} \leq \cdots \leq \varepsilon_{m}$ be the eigenvalues of $-\widehat{W}_{12}^{2}$ on $E_{k}$, then we may write $\left(\alpha-\mu_{1}\right)\left(\alpha-\mu_{2}\right) /\left\|W_{12}\right\|^{2}=\varepsilon_{r}$ for some $r$ 
and hence $\alpha^{2}-\left(\mu_{1}+\mu_{2}\right) \alpha+\mu_{1} \mu_{2}-\left\|W_{12}\right\|^{2} \varepsilon_{r}=0$. By the quadratic formula

$$
2 \alpha=\left(\mu_{1}+\mu_{2}\right) \pm \sqrt{\left(\mu_{1}+\mu_{2}\right)^{2}-4\left(\mu_{1} \mu_{2}-\left\|W_{12}\right\|^{2} \varepsilon_{r}\right)} .
$$

It is a necessary condition for stability that $\mu_{1}, \mu_{2} \geq 0$. If we take the plus sign in the quadratic formula along with the condition that $\mu_{1}, \mu_{2} \geq 0$ then $\alpha \geq 0$. Consider the case with the minus sign. By Theorem 3.4 , in order to have stability we must have

$$
2 \lambda_{k}+\left(\mu_{1}+\mu_{2}\right)-\sqrt{\left(\mu_{1}+\mu_{2}\right)^{2}-4\left(\mu_{1} \mu_{2}-\left\|W_{12}\right\|^{2} \varepsilon_{r}\right)} \geq 0,
$$

which implies that

$$
\lambda_{k}^{2}+\lambda_{k}\left(\mu_{1}+\mu_{2}\right)+\mu_{1} \mu_{2} \geq\left\|W_{12}\right\|^{2} \varepsilon_{r} .
$$

If $\varepsilon_{r}=0$ this condition is clearly satisfied, so suppose $\varepsilon_{r}>0$. Then we have

$$
\left\|W_{12}\right\|^{2} \leq \frac{\left(\lambda_{k}+\mu_{1}\right)\left(\lambda_{k}+\mu_{2}\right)}{\varepsilon_{r}} .
$$

Conversely, suppose that for some $k$ the operator $-\left.\widehat{W}_{12}^{2}\right|_{E_{k}}$ has an eigenvalue $\varepsilon_{r}$ such that

$$
\left\|W_{12}\right\|^{2}>\frac{\left(\lambda_{k}+\mu_{1}\right)\left(\lambda_{k}+\mu_{2}\right)}{\varepsilon_{r}} .
$$

Let $\varphi \in C^{\infty}(P)$ be such that $\Delta \varphi=\lambda_{k} \varphi$ and $-W_{12}^{2} \varphi=\left\|W_{12}\right\|^{2} \varepsilon_{r} \varphi$ with $\int_{P} \varphi^{2}=1$. Observe that for any $W=A^{1} W_{1}+A^{2} W_{2}$ with $W \in E_{k}$ we have

$$
\int_{P}\langle J W, W\rangle=\int_{P}\left(\lambda_{k}+\mu_{1}\right)\left(A^{1}\right)^{2}-2 A^{1} W_{12} A^{2}+\left(\lambda_{k}+\mu_{2}\right)\left(A^{2}\right)^{2} .
$$

Let $A^{1}=\left(\lambda_{k}+\mu_{2}\right)^{1 / 2} \varphi$ and

$$
A^{2}=\left(\lambda_{k}+\mu_{1}-\frac{\varepsilon_{r}\left\|W_{12}\right\|^{2}}{\lambda_{k}+\mu_{2}}\right)^{1 / 2} \varphi-\left(\lambda_{k}+\mu_{2}\right)^{-1 / 2} W_{12} \varphi .
$$

Then $\left(A^{1}\right)^{2}=\left(\lambda_{k}+\mu_{2}\right) \varphi^{2}$ and

$$
\begin{aligned}
\left(A^{2}\right)^{2}= & \left(\lambda_{k}+\mu_{1}-\frac{\varepsilon_{r}\left\|W_{12}\right\|^{2}}{\lambda_{k}+\mu_{2}}\right) \varphi^{2}-2\left(\lambda_{k}+\mu_{1}-\frac{\varepsilon_{r}\left\|W_{12}\right\|^{2}}{\lambda_{k}+\mu_{2}}\right)^{1 / 2} \\
& \cdot\left(\lambda_{k}+\mu_{2}\right)^{-1 / 2} \varphi W_{12} \varphi+\left(\lambda_{k}+\mu_{2}\right)^{-1}\left(W_{12} \varphi\right)^{2}-2 A^{1} W_{12} A^{2} \\
= & -2\left(\lambda_{k}+\mu_{1}-\frac{\varepsilon_{r}\left\|W_{12}\right\|^{2}}{\lambda_{k}+\mu_{2}}\right)^{1 / 2}\left(\lambda_{k}+\mu_{2}\right)^{1 / 2} \varphi W_{12} \varphi+2 \varphi W_{12}^{2} \varphi .
\end{aligned}
$$


By Lemma 3.1 $W_{12}$ is a skew-symmetric operator, so it follows that

$$
\int_{P}\left(W_{12} \varphi\right)^{2}=-\int_{P} \varphi W_{12}^{2} \varphi=\int_{P} \varepsilon_{r}\left\|W_{12}\right\|^{2} \varphi^{2}=\varepsilon_{r}\left\|W_{12}\right\|^{2}
$$

and $\int_{P} \varphi W_{12} \varphi=0$. Therefore, $\int_{P}\left(A^{1}\right)^{2}=\lambda_{k}+\mu_{2}, \int_{P}\left(A^{2}\right)^{2}=\lambda_{k}+\mu_{1}$, and $-2 \int_{P} A^{1} W_{12} A^{2}=-2\left\|W_{12}\right\|^{2} \varepsilon_{r}$ so

$$
\int_{P}\langle J W, W\rangle=2\left(\lambda_{k}+\mu_{1}\right)\left(\lambda_{k}+\mu_{2}\right)-2\left\|W_{12}\right\|^{2} \varepsilon_{r}<0 .
$$

Hence $P$ is unstable.

EXAMPLE. Consider the case where the orbits of $G$ are isometric to constant curvature spheres. In the case where the dimension of the orbit is even, $W_{12}=0$ since even dimensional spheres do not admit non-vanishing vector fields. This implies that a minimal codimension two orbit is stable if and only if $\mu_{1}, \mu_{2} \geq 0$.

Now consider the case where $G$ is the unitary group $U(n)$ and the codimension two minimal orbit $P$ is equivariantly isometric to $S^{2 n-1} \subset C^{n}$ embedded in the standard way. The flow of an invariant Killing vector field $X$ on $S^{2 n-1}, n \geq 1$, must commute with the action of $U(n)$, and hence must be the restriction to $S^{2 n-1}$ of the action of $S^{1}$ on $C^{n}$ by scalar multiplication. The trajectories of $X$ are geodesics of length $2 \pi$.

The $k$ th eigenspace of the laplacian on $S^{2 n-1}$ consists of the restrictions to $S^{2 n-1}$ of homogeneous harmonic polynomials of degree $k$ in $z_{1}, \ldots, z_{n}, \bar{z}_{1}, \ldots, \bar{z}_{n}$. Simultaneous eigenfunctions of $X^{2}$ and $\Delta$ are restrictions to $S^{2 n-1}$ of linear combinations of the same degree $\alpha$ in $z_{1}, \ldots, z_{n}$ and same degree $\beta$ in $\bar{z}_{1}, \ldots, \bar{z}_{n}$ with $\alpha+\beta=k$. Clearly the largest eigenvalue of $-X^{2}$ on the $k$ th eigenspace of $\Delta$ is $k^{2}$. The $k$ th eigenvalue of $\Delta$ on $S^{2 n-1}$ is $\lambda_{k}=k(k+2 n-2)$. By Theorem $3.7 P$ is stable if and only if

$$
\left\|W_{12}\right\|^{2}=\inf _{k>0}\left(\frac{\left[k^{2}+k(2 n-2)+\mu_{1}\right]\left[k^{2}+k(2 n-2)+\mu_{2}\right]}{k^{2}}\right) .
$$

Define $\lambda=\min \left\{\mu_{1}, \mu_{2}\right\}$. Then the right-hand side of the above formula is at least

$$
\inf _{k>0}\left(\frac{k^{2}+k(2 n-2)+\lambda}{k}\right)^{2} .
$$

Because the function $f(x)=\left(x^{2}+(2 n-1) x+\lambda\right) / x$ achieves its absolute minimum over $[0, \infty)$ at $x=\sqrt{\lambda}$, we see that $P$ is stable 
if $\left\|W_{12}\right\| \leq 2 \sqrt{\lambda}+2 n-2$. Next define $\mu=\frac{1}{2}\left(\mu_{1}+\mu_{2}\right)$. Then

$$
\begin{gathered}
\frac{\left[k^{2}+(2 n-2) k+\mu_{1}\right]\left[k^{2}+(2 n-2) k+\mu_{2}\right]}{k^{2}} \\
=\frac{\left(k^{2}+(2 n-2) k+\mu\right)^{2}-\frac{1}{4}\left(\mu_{1}-\mu_{2}\right)}{k^{2}} .
\end{gathered}
$$

If we let $k=1$, then we see that $P$ is unstable if

$$
\left\|W_{12}\right\|^{2}>(2 n-1+\mu)^{2}-\frac{1}{4}\left(\mu_{1}-\mu\right)^{2} .
$$

We note that according to Brothers' results [Br2], $P$ is unstable if $\left\|W_{12}\right\|^{2}>(2 n-1+\mu)^{2}$.

REMARKS. In the case where the integral curves of $W_{12}$ are circles of length $L$, the eigenvalues of $-W_{12}^{2}$ must be of the form $2 \pi k / L$ for some integer $k$.

Brothers' condition for stability [Br1] requires that the hessian of the volume function have no non-negative eigenvalues along with the condition $\left\|W_{12}\right\|^{2} \leq 2 \pi / L$ for codimension two principal orbits. In order to compare this result with ours, we make use of the fact $\lambda_{k} \geq \sigma_{k}$ for all $k$ [B1]. Observe that

$$
\frac{\left(\lambda_{k}+\mu_{1}\right)\left(\lambda_{k}+\mu_{2}\right)}{\sigma_{k}} \geq \frac{\left(\sigma_{k}+\mu_{1}\right)\left(\sigma_{k}+\mu_{2}\right)}{\sigma_{k}}=\sigma_{k}+\mu_{1}+\mu_{2}+\frac{\mu_{1} \mu_{2}}{\sigma_{k}} .
$$

By the above remark, we see that $\sigma_{k} \geq 2 \pi / L$ for all $k$, so we conclude that

$$
\frac{\left(\lambda_{k}+\mu_{1}\right)\left(\lambda_{k}+\mu_{2}\right)}{\sigma_{k}} \geq \frac{2 \pi}{L}+\mu_{1}+\mu_{2}+\frac{\mu_{1} \mu_{2}}{\sigma_{k}} .
$$

Hence, our theorem gives stability for larger $\left\|W_{12}\right\|$.

4. Exceptional orbits. Let $E$ be an exceptional orbit with isotropy group $G_{x_{0}}$. E has a tubular neighborhood $N$ which is equivariantly diffeomorphic to $G \times_{G_{x_{0}}} V$ where $V$ is a real vector with dimension equal to the codimension of $E$ in $N$. There exists a subgroup $H_{x_{0}} \subset G_{x_{0}}$ with a finite index in $G_{x_{0}}$ such that $H_{x_{0}}$ is conjugate to the isotropy group of every principal orbit. $H_{x_{0}}$ is normal in $G_{x_{0}}$ and is precisely the non-effective part of $G_{x_{0}}$ on $V$ [B].

Consider the space $\tilde{N}=G / H_{x_{0}} \times V$ and the map $\pi: \tilde{N} \rightarrow N$ defined by $\pi\left(g H_{x_{0}}, v\right)=[g, v]$ where $[g, v]$ is the equivalence class of $(g, v)$ under the action of $H_{x_{0}}$ given by $h(g, v)=\left(g h^{-1}, h v\right)$. This map is well defined because $h V=V$ and hence $[g h, v]=[g, v]$ for $h \in H_{x_{0}}$. It also follows easily that $\pi$ is equivariant. 
Observe that $\pi^{-1}\{[g, v]\}=\left\{\left(g y H_{x_{0}}, y^{-1} v\right): y \in G_{x_{0}}\right\}$. This observation combined with the fact that $H_{x_{0}}$ acts trivially on $V$ implies that $\pi^{-1}\{x\}$ has the same cardinality as $G_{x_{0}} / H_{x_{0}}$ for all $x \in N$. $G_{x_{0}} / H_{x_{0}}$ is a finite group since $H_{x_{0}}$ is normal in $G_{x_{0}}$. We define a right action of $G_{x_{0}} / H_{x_{0}}$ on $\tilde{N}$ by $\left(g H_{x_{0}}, v\right) g^{\prime} H_{x_{0}} \equiv\left(g g^{\prime} H_{x_{0}},\left(g^{\prime}\right)^{-1} v\right)$ for $\left(g H_{x_{0}}, v\right) \in \widetilde{N}$ and $g^{\prime} H_{x_{0}} \in G_{x_{0}} / H_{x_{0}}$. The action of this group is transitive on the fibers of $\pi$ and the cardinality of each fiber is the same as $G_{x_{0}} / H_{x_{0}}$. Hence $G_{x_{0}} / H_{x_{0}}$ acts freely and discontinuously, which implies that $\pi$ is a covering map whose group of deck transformations is $G_{x_{0}} / H_{x_{0}}$.

Equip $\widetilde{N}$ with the metric such that $\pi$ is a local isometry. There is an action of $G$ on $\tilde{N}$ given by $g\left(g^{\prime} H_{x_{0}}, v\right)=\left(g g^{\prime} H_{x_{0}}, v\right)$. All of the orbits of $\tilde{N}$ are principal and the orbit $P=\left\{\left(g H_{x_{0}}, 0\right): g \in G\right\}$ is an $m$-fold covering of $E$ under the map $\pi$, where $m=\operatorname{card}\left(G_{x_{0}} / H_{x_{0}}\right)$.

EXAMPLE. Consider the Möbius strip $M=S^{1} \times{ }_{Z_{2}} R$, where $Z_{2}=$ $\{1,-1\}$ acts on $R$ by multiplication. The action of $S^{1}$ on $M$ is given by $F: S^{1} \times M \rightarrow M$ with $F\left(e^{i \alpha},\left[e^{i \theta}, r\right]\right)=\left[e^{i(\alpha+\theta)}, r\right]$. The center circle has length $\pi$ and is given by $E=\left\{\left[e^{i \theta}, 0\right]: 0 \leq \theta \leq 2 \pi\right\}$, and it is an exceptional orbit with $G_{x}=Z_{2}$ for all $x$. All of the other orbits have length $2 \pi$ and are principal with isotropy group given by $\{1\}$. In this case $\tilde{N}=S^{1} \times R$ which is a cylinder that is the oriented double cover of $M$.

Suppose that $W$ is a smooth vector field defined on the orbit $E$ and normal to $E$. Let $\widetilde{W}$ be the lift of $W$ to $P=\pi^{-1}(E)$ in $\widetilde{N}$.

\section{LEMMA 4.1. $m \delta_{E}^{(2)}(W)=\delta_{P}^{(2)}(\widetilde{W})$.}

Proof. Extend $W$ to a neighborhood of $E$ and lift to $\widetilde{W}$. Let $E_{t}$ be the image of $E$ under the flow of $W$ and $P_{t}$ be the image of $P$ under the flow $\tilde{\phi}_{t}$ determined by $\widetilde{W}$. If we take $t$ small enough and use the fact $\phi_{t}=\pi \circ \tilde{\phi}_{t}$, we see that $P_{t}$ is a cover of $E_{t}$ with covering map $\left.\pi\right|_{P_{t}}$. If we let $A(t)$ be the volume of $E_{t}$ and $\widetilde{A}(t)$ be the volume of $P_{t}$, then $\widetilde{A}(t)=m A(t)$ since $P_{t}$ is an $m$-fold cover of $E_{t}$. Thus $\widetilde{A}^{\prime \prime}(0)=m A^{\prime \prime}(0)$.

COROLLARY 4.2. If for every normal vector field $W$ on $E$ we have $\delta_{P}^{(2)}(\widetilde{W}) \geq 0$, then $E$ is stable. By the above arguments we see that we have reduced the problem of the stability of an exceptional orbit to the stability of a principal orbit under a restricted class of vector fields, 
namely the ones that are lifts. We will exploit special properties of the components of the lift of a vector field, along with the second variation formula for principal orbits, to find sufficient conditions for stability.

Let $G_{x_{0}}$ be the isotropy group of an exceptional orbit $E$ and let $H_{x_{0}}$ be the conjugate of some isotropy group of a principal orbit such that $H_{x_{0}} \subset G_{x_{0}}$. Consider the linear action of the isotropy group on the normal space $V_{x_{0}}$ to $E$ at $x_{0}$. This gives a linear representation of $G_{x_{0}}$ on $V_{x_{0}}$ and the kernel of this representation is $H_{x_{0}}$. For an arbitrary point $x \in E$, the representation of $G_{x}$ on $V_{x}$ is clearly equivalent to the representation of $G_{x_{0}}$ on $V_{x_{0}}$. Hence we may speak of the representation of an isotropy group of $E$ without reference to a point.

Proposition 4.3. Let $G(y)$ be an orbit in $N$. Then $\pi^{-1}\{G(y)\}$ consists of $n_{y}$ disjoint orbits where $n_{y}=\operatorname{card}\left(G_{x_{0}} / G_{y}\right)$. If $P$ is one of these orbits then $\left.\pi\right|_{P}: P \rightarrow G(y)$ is an $m_{y}$-fold covering map where $m_{y}=\operatorname{card}\left(G_{y} / H_{x_{0}}\right)$.

Proof. Without loss of generality we may assume that $y=[e, v]$. For $g \in G_{y}$ we have $[e, v]=g[e, v]=[g, v]$. Hence there must be a $g^{\prime} \in G_{x_{0}}$ such that $g g^{\prime}=e$ and $\left(g^{\prime}\right)^{-1} v=v$. Consequently, $G_{y} \subset G_{x_{0}}$ and $g^{\prime} v=v$ for $g^{\prime} \in G_{y}$. Consider the action of $G_{x_{0}}$ on $V$ and let $\left(G_{x_{0}}\right)_{v}$ be the isotropy of $v$. For $g \in\left(G_{x_{0}}\right)_{v}$ we have $g y=g[e, v]=[g, v]=[e, g v]=[e, v]=y$. So $\left(G_{x_{0}}\right)_{v} \subset G_{y}$ and as was seen above, $G_{y}$ fixes $v$. We conclude that $G_{y}=\left(G_{x_{0}}\right)_{v}$. Let $\left\{v_{1}, \cdots, v_{r}\right\}$ be the orbit of $v$ under the action of $G_{x_{0}}$ where $r=\operatorname{card}\left(G_{x_{0}} / G_{y}\right)$. Let $C_{1}, \ldots, C_{r}$ denote the left cosets of $G_{y}$ in $G_{x_{0}}$ and observe that $\pi^{-1}\{y\}=\pi^{-1}\{[e, v]\}=\left\{\left(g^{-1} H_{x_{0}}, g v\right): g \in G_{x_{0}}\right\}=$ $\bigcup_{i=1}^{r}\left\{\left(g^{-1} H_{x_{0}}, v_{i}\right): g \in C_{i}\right\}$. The sets $\left\{\left(g^{-1} H_{x_{0}}, v_{i}\right): g \in C_{i}\right\}, i=$ $1, \ldots, r$ lie on distinct orbits which means that there are $r=n_{y}$ orbits in $\pi^{-1}\{G(y)\}$. The principal orbit $P=\left\{\left(g H_{x_{0}}, v_{i}\right): g \in G\right\}$ is then a cover of the orbit $G(y)$.

REMARK. This proposition may be illustrated by considering the previous example of the Möbius strip. The inverse image under $\pi$ of the exceptional orbit (center circle) of the Möbius strip is a circle that is a two-fold cover and the inverse image under $\pi$ of any principal orbit is two disjoint circles each of which is a one-fold cover.

We have assumed that $N=G \times_{G_{x_{0}}} V$ where $G_{x_{0}}$ is the isotropy group of an exceptional orbit $E$. It follows that all orbits in $N$ are 
principal or exceptional. We now define a volume function $\nu$ on $N$ that is related to the volume function $\tilde{\nu}$ on $\tilde{N}$. Define $\nu: N \rightarrow R$ by $\nu(x)=m_{x} \mathscr{H}^{r}(G(x))$, where $\mathscr{H}^{r}$ is the Hausdorff $r$-measure induced by the metric and $r$ is the dimension of a principal orbit. We have

\section{LEMMA 4.4. $\tilde{\nu}=\nu \circ \pi$.}

Proof. $\tilde{\nu}(z)$ is the volume of the principal orbit $P$ through $z \in \tilde{N}$. By Proposition 4.3 $P$ is an $m_{\pi(z)}$-fold cover of the orbit $\pi(P)$. Since $\pi$ is a local isometry, we see that the volume of $P$ must be $m_{\pi(z)}$ times the volume of $G(\pi(z))$.

It is now clear that if $\nu$ is critical on the orbit $E$, then $\tilde{\nu}$ has a critical point on $P$. Hence the hessian of $\tilde{\nu}$ is defined.

We now develop some of the properties of the components of the lift of a vector field. Suppose that $\left\{W_{1}, \ldots, W_{n}\right\}$ is an orthonormal $G$-invariant frame field for the normal bundle of $P$ and $\pi(p)=x$ with $p \in P$ and $x \in E$. Then $\left\{\pi_{*} W_{1}(p), \ldots, \pi_{*} W_{n}(p)\right\}$ is an orthonormal frame field for the normal space $V$ at $x$. We denote by $\left\{T_{i j}\right\}, i, j=$ $1, \ldots, n$, the matrices of the linear representation of $G_{x}$ with respect to the basis $\left\{\pi_{*} W_{1}(p), \ldots, \pi_{*} W_{n}(p)\right\}$.

LEMMA 4.5. Let $W$ be a normal vector field on $E$ with lift $\widetilde{W}=$ $\sum_{k} A^{k} W_{k}$ to $P$ and let $\pi\left(p^{\prime}\right)=\pi(p)=x$. Then

$$
A^{i}\left(p^{\prime}\right)=\sum_{j} T_{i j}(g) A^{j}(p)
$$

with $p^{\prime}=g p, g \in G_{x}$ and $x \in E$.

Proof. Since $G_{x}$ acts transitively on $\pi^{-1}\{x\}$, there exists $g \in G_{x}$ such that $p^{\prime}=g p$ and $W\left(p^{\prime}\right)=g_{*} W_{k}(p), k=1, \ldots, n$. By definition $A^{i}\left(p^{\prime}\right)=\left\langle\widetilde{W}\left(p^{\prime}\right), W_{i}\left(p^{\prime}\right)\right\rangle$. The result then follows by applying the facts that $\pi$ is an equivariant isometry and $\widetilde{W}$ is a lift.

LEMMA 4.6. Let $E$ be an isolated exceptional orbit with isotropy group $G_{x_{0}}$. Then $G_{x_{0}}$ does not fix any vector normal to $E$ at $x$.

Proof. Suppose there were a vector $v_{0}$ such that $G_{x_{0}} v_{0}=v_{0}$. Then we would have a one parameter family of exceptional orbits of the same type as $E$ in the tubular neighborhood $G \times_{G_{x_{0}}} V$ given by $\{G([e, t v]):-1 \leq t \leq 1\}$. But this contradicts the fact that $E$ is isolated. 
COROLLARY 4.7. Let $E$ be an isolated exceptional orbit and $\widetilde{W}=$ $\sum_{i} A^{i} W_{i}$ be the lift of some vector field $W$. Then $\int_{P} A^{i}=0$ for all $i$.

Proof. Let $x \in E$ and recall that $\pi^{-1}\{x\}$ can be canonically identified with the set $G_{x} / H_{x}$. Fix $p \in \pi^{-1}\{x\}$. Then we have

$$
\sum_{\pi^{-1}\{x\}} A^{i}=\sum_{\tilde{g} \in G_{x} / H_{x}} A^{i}(\tilde{g} p) \text {. }
$$

However, by Lemma 4.5 we have $A^{i}(\tilde{g} p)=\sum_{j} T_{i j}(\tilde{g}) A^{j}(p)$. Observe that the sum of the linear transformations of the representation of a finite group must be zero unless there is a vector fixed by all the transformations of the representation. Hence by Lemma 4.5 we see that $\sum_{\pi^{-1}(x)} A^{i}=0$. But

$$
\int_{P} A^{i}=\int_{E} \sum_{\pi^{-1}(x)} A^{i} d x
$$

Let $E$ be a minimal exceptional orbit in $N$ covered by the principal orbit $P$ in $\tilde{N}$. We say that the normal distribution to $E$ is involutive if there exist orthonormal vector fields $V_{1}, \ldots, V_{n}$, defined on a neighborhood $U$ of $x \in E$ in $N$ where $n$ is the codimension of $E$ in $N$, such that the projection on $E$ of $\left[V_{i}, V_{j}\right]$ is zero for all $i, j$ for every $x$. See $[B r 1]$. Note that since a tubular neighborhood of $P$ is locally isometric to a tubular neighborhood of $E$, it follows that the normal distribution to $E$ is involutive if and only if the normal distribution to $P$ is involutive. Because $P$ is locally isometric to $E$, we can define $\pi_{*} W_{1}, \ldots, \pi_{*} W_{n}$ locally on $E$. Since $\nu$ has a critical point on $E$, the hessian of $\nu$ on $E$ satisfies $H\left(\pi_{*} W_{i}, \pi_{*} W_{j}\right)=\widetilde{H}\left(W_{i}, W_{j}\right)$ where $\widetilde{H}$ is the hessian of $\tilde{\nu}$ on $P$. Since the hessian of a function at a critical point $p \in \widetilde{N}$ is a map of $T_{p}(\widetilde{N}) \times T_{p}(\widetilde{N})$, we can conclude that for $v, w \in T_{p}(\widetilde{N})$, the hessian $\widetilde{H}$ of $\tilde{\nu}$ on $P$ satisfies $\widetilde{H}(v, w)=H\left(\pi_{*} v, \pi_{*} w\right)$. In particular, it follows that $H$ and $\widetilde{H}$ have the same eigenvalues which are constant because $\widetilde{H}$ is $G$ invariant.

THEOREM 4.8. Let $E$ be an isolated exceptional orbit with the normal distribution to $E$ involutive. If

$$
\mu_{k} \geq-\nu(x) \lambda_{1}
$$

for $k=1, \ldots, n$, where $\lambda_{1}$ is the first eigenvalue of $\Delta$ on $P$, and the $\mu_{k}$ are the eigenvalues of $H$ with $x \in E$, then $E$ is minimal and stable. 
Proof. $E$ is stable if $P$ is stable under all lifts $\widetilde{W}$. Because the normal distribution to $E$ is involutive, we conclude that the $W_{i k}=$ $\left[W_{i}, W_{k}\right]$ for $i, k=1, \ldots, n$ all vanish on $P$. Then by the second variation formula for principal orbits we have

$$
\delta_{P}^{(2)}(\widetilde{W})=\int_{P} \sum_{i=1}^{n} \frac{1}{\tilde{\nu}(p)} \mu_{i}\left(A^{i}\right)^{2}+\left\|d A^{i}\right\|^{2}
$$

where $\widetilde{W}=\sum_{i=1}^{n} A^{i} W_{i}$ and $\mu_{1}, \ldots, \mu_{n}$ are the eigenvalues of the hessian $\widetilde{H}$ on $P$ and hence the eigenvalues of $H$ on $E$. But $\int_{P} A^{i}=$ 0 (Corollary 4.7) so we may apply the Poincare inequality and use the fact that $\tilde{\nu}(p)=\nu(x)$ to infer that $E$ is stable if $\mu_{i} \geq-\nu(x) \lambda_{1}$ for $i=1, \ldots, n$.

REMARK. Compare this with the case of a principal orbit where stability implies $\mu_{i} \geq 0$ for all $i$.

LEMMA 4.9. Let $G$ be a finite group with an irreducible orthogonal representation on an $n$-dimensional real inner product space $V$. Let $\{U(g): g \in G\}$ be the matrices of this representation and $M: V \rightarrow V$ a symmetric endomorphism. Then

$$
\sum_{g \in G} U^{-1}(g) M U(g)=\left(\frac{1}{n} \operatorname{tr} M\right) I
$$

where $I$ is the identity transformation on $V$.

Proof. This follows from the basic Schurs' lemma in representation theory [BD].

Theorem 4.10. Let $E$ be an isolated exceptional orbit. Suppose that the normal distribution to $E$ is involutive and the representation of the isotropy group on the normal space is irreducible. If $\operatorname{tr} H \geq 0$, then $E$ is stable.

Proof. Let $W$ be a normal vector field on $E$ with lift $\widetilde{W}=\sum_{i=1}^{n} A^{i} W_{i}$ to $P$. Consider any $x \in E$, and let $H_{x} \subset G_{x}$ be the isotropy group of some principal orbit. Let $p \in \pi^{-1}\{x\}$ with $h p=p$ for all $h \in H_{x}$. Then

$$
\sum_{\pi^{-1}\{x\}} \sum_{i, j} H_{i j} A^{i} A^{j}=\sum_{\tilde{g} \in G_{x} / H_{x}} \sum_{i, j} H_{i j} A^{i}(\tilde{g} p) A^{j}(\tilde{g} p) .
$$


By Lemma 4.5 we infer that the right-hand side is the same as

$$
\sum_{r, k} A^{k}(p) A^{r}(p) \sum_{\tilde{g} \in G_{x} / H_{x}} \sum_{i, j} T_{i k}(\tilde{g}) H_{i j} T_{j r}(\tilde{g}) .
$$

Applying Lemma 4.9, we see that this last sum is

$$
\frac{1}{n}\left(\sum_{k}\left(A^{k}(p)\right)^{2}\right) \operatorname{tr} H
$$

But

$$
\int_{P} \sum_{i, j} H_{i j} A^{i} A^{j}=\int_{E} \sum_{\pi^{-1}(x)} \sum_{i, j} A^{i} A^{j} d x .
$$

From this computation we see that $\delta_{P}^{(2)}(\widetilde{W}) \geq 0$ if $\operatorname{tr} H \geq 0$.

5. Examples. Consider the standard $m$-sphere

$$
S^{m}=\left\{\left(x_{0}, \ldots, x_{m}\right): \sum_{k=0}^{m} x_{k}^{2}=1\right\} .
$$

For $n<m$ we have the standard inclusion $S^{n} \hookrightarrow S^{m}$ where $S^{n}=$ $\left\{\left(x_{0}, \ldots, x_{n}, 0, \ldots, 0\right): \sum_{k=0}^{n} x_{k}^{2}=1\right\}$. Consider a tubular neighborhood of $S^{n}$ in $S^{m}$ given by

$$
\widetilde{N}=\left\{\left(x_{0}, \ldots, x_{m}\right) \in S^{m}:\left(x_{0}, \ldots, x_{n}\right) \neq 0\right\} .
$$

Suppose $G$ acts on $S^{m}$ such that the orbits in $\tilde{N}$ are all principal of the form

$$
P_{\vec{u}}=\left\{\left(x_{0}, \ldots, x_{n}, u_{1}, \ldots, u_{m-n}\right): \sum_{k=0}^{n} x_{k}^{2}=1-\|\vec{u}\|^{2}\right\}
$$

where $\vec{u}=\left(u_{1}, \ldots, u_{m-n}\right)$ satisfies $\|\vec{u}\|<1$. Each $P_{\vec{u}}$ is diffeomorphic to $S^{n}$ and $P_{\overrightarrow{0}}$ is the standard $S^{n}$ in $S^{m}$.

Let $D$ be a finite group which acts freely on $S^{m}$ on the right and commutes with the action of $G$. It follows that $G$ acts on $S^{m} / D$ and the canonical map $\pi: S^{m} \rightarrow S^{m} / D$ is equivariant. Furthermore, suppose $\pi\left(P_{\vec{u}}\right)$ is an exceptional orbit for $\vec{u}=0$ and a prinicpal orbit $\vec{u} \neq 0$. Let $x_{0} \in E \equiv \pi\left(P_{\overrightarrow{0}}\right)$. Since $E$ is exceptional, it has a tubular neighborhood $N$ equivariantly diffeomorphic to $G \times_{G_{x_{0}}} V$. We suppose that $G_{x_{0}} / H_{x_{0}}$ is isomorphic to $D$ and that the action of $G_{x_{0}} / H_{x_{0}}$ on $\pi^{-1}(N)$ is identical to the action of $D . E$ is clearly a 
minimal submanifold since it is isolated. We now have:

Proposition 5.1. E is stable minimal submanifold of $S^{m} / D$.

Proof. Clearly, each principal orbit $P_{\vec{u}}$ intersects the $(m-n)$-sphere of radius 1 given by

$\left\{\left(x_{0}, \ldots, x_{n+1}, u_{1}, \ldots, u_{m-n}\right): x_{0}^{2}+\|u\|^{2}=1, x_{1}=\cdots=x_{n+1}=0\right\}$

at the points $\left( \pm\left(1-\|u\|^{2}\right)^{1 / 2}, 0, \ldots, 0, u_{1}, \ldots, u_{m-n}\right)$. Furthermore, this $(m-n)$-sphere is perpendicular to the orbits. By the Frobenius integrability condition, we see that the normal distribution to $E$ is involutive. The first eigenvalue of $\Delta$ on $S^{n}$ is $n$. Let us now compute the hessian of the volume function at the principal orbit $P_{\overrightarrow{0}}=S^{n}$. Note that $P$ is located at the intersection of exactly $m-n$ mutually orthogonal isometric copies of $S^{n+1}$. Let $\theta$ be the angle between a radius of $S^{n+1}$ and a radius drawn to its north pole. Then $\tilde{\nu}(\theta)=\omega_{n} \sin ^{n} \theta$ where $\omega_{n}$ is the volume of the unit $n$-sphere. It easily follows that the hessian has eigenvalue 0 along the orbit $P_{\overrightarrow{0}}$ and eigenvalue $-n \omega_{n}$ for any direction normal to the orbit. Hence $E$ is stable by Theorem 4.8 .

COROLlaRY 5.2. The standard inclusion of $R P^{n}$ in $R P^{m}$ is stable for $n<m$.

Proof. In Proposition 5.1 let $G=\mathrm{SO}(n+1)$ and $D=Z_{2} . \mathrm{SO}(n+1)$ acts by matrix multiplication on the first $(n+1)$ coordinates of a point in $S^{m}$ considered as a column vector. The action of $D$ identifies a point with its anitpodal point. Clearly the action of $\operatorname{SO}(n+1)$ commutes with the action $D, S^{m} / D \approx R P^{m}$, and $E=R P^{n}$.

REMARK. Actually, $R P^{n}$ is area minimizing in $R P^{m}[F]$.

Next consider $S^{2 m-1}$ respresented by the set

$$
\left\{\left(z_{1}, \ldots, z_{m}\right): \sum_{k=1}^{m}\left|z_{k}\right|^{2}=1 ;\left(z_{1}, \ldots, z_{m}\right) \in C^{m}\right\} \text {. }
$$

Let $U(n)$ act on $S^{2 m-1}$ by matrix multiplication on the first $n$ entries considered as a column vector. Let $\left(q_{1}, \ldots, q_{m}\right)$ have positive integer coordinates such that $\left(p, q_{i}\right)=1$ for $i=1, \ldots, m$. The lens space $L\left(p ; q_{1}, \ldots, q_{m}\right)$ is $S^{2 m-1}$ modulo the action of $D=Z_{p}$ given by $l\left(z_{1}, \ldots, z_{m}\right)=\left(z_{1} e^{2 \pi i q_{1} l / p}, \ldots, z_{m} e^{2 \pi i q_{m} l / p}\right), l=0, \ldots, p-1$. If $q_{1}=q_{2}=\cdots=q_{n}$, then the action of $Z_{p}$ on $S^{2 m-1}$ commutes with 
the action of $U(n)$. We also see that $\pi\left(S^{2 n-1}\right)$ is $L\left(p ; q_{1}, \ldots, q_{n}\right)$ which is an exceptional orbit in $L\left(p ; q_{1}, \ldots, q_{m}\right)$. Hence by Proposition 5.1 we have

Corollary 5.3. Suppose that $\left(p, q_{i}\right)=1$ for $i=1, \ldots, m$ and $q_{1}=q_{2}=\cdots=q_{n}$ with $n<m$. Then $L\left(p ; q_{1}, \ldots, q_{n}\right)$ is a stable minimal submanifold of $L\left(p ; q_{1}, \ldots, q_{m}\right)$.

Finally, consider $S^{4 m-1}$ represented by the set

$$
\left\{\left(q_{1}, \ldots, q_{m}\right): \sum_{k=1}^{m}\left\|q_{k}\right\|^{2}=1 ;\left(q_{1}, \ldots, q_{m}\right) \in H^{m}\right\} .
$$

There is a natural action of $G=\operatorname{Sp}(n)$ on $S^{4 m-1}$ by matrix multiplication on the first $n$ coordinates considered as a column vector.

Let $D$ be a finite subgroup of the unit quaternions and define a right action of $D$ on $S^{4 m-1}$ by $\left(q_{1}, \ldots, q_{m}\right) q \equiv\left(q_{1} q, \ldots, q_{m} q\right), q \in D$. We denote $S^{4 m-1} / D$ by $S F(D, m)$ and is known as the quaternionic space-form defined by the action of $D$ on $S^{4 m-1}$. The action of $\mathrm{Sp}(n)$ commutes with the action of $D$ and $\pi\left(S^{4 n-1}\right)=S F(D, n)$ is an exceptional orbit in $S F(D, m)$ for $n<m$. Hence by Proposition 5.1 we have

COROllary 5.4. $S F(D, n)$ is a stable minimal submanifold of $S F(D, m)$ for $n<m$.

\section{REFERENCES}

[B1] D. Bleeker, The spectrum of a Riemannian manifold with a unit Killing vector field, Trans. Amer. Math. Soc., 275 (1983), 409-416.

[B] G. E. Bredon, Introduction To Transformation Groups, Academic Press, New York and London (1972).

[BD] T. Bröker and T. tom Dieck, Representations of Compact Lie Groups, SpringerVerlag, New York, (1985).

[Br1] J. Brothers, Stability of minimal orbits, Trans. Amer. Math. Soc., 294 (1986), 537-552.

[Br2] _- Second Variation Estimates for Minimal Orbits, Geometric Measure Theory and the Calculus of Variations (Proceedings of Symposia in Pure Mathematics, volume 44), (1984), 139-149.

[F] A. T. Fomenko, Minimal compacta in Riemannian manifolds and Reifenberg's conjecture, Math. USSR Izvestija, (1972).

[HL] W. Y. Hsiang and H. B. Lawson, Minimal submanifolds of low cohomogeneity, J. Differential Geom., 5 (1971), 1-38. 
[P] W. A. Poor, Differential Geometric Structures, McGraw-Hill, Inc. New York, 1981.

[W] F. W. Warner, Foundations of Differentiable Manifolds and Lie Groups, Scott, Foresman and Company, Glenview, Illinois (1971).

Received March 12, 1991.

West Chester UNiversity

West Chester, PA 19383 


\title{
PACIFIC JOURNAL OF MATHEMATICS
}

Founded by

\author{
E. F. BECKENBACH (1906-1982) F. WolF (1904-1989) \\ EDITORS
}

\author{
V. S. VARADARAJAN \\ (Managing Editor) \\ University of California \\ Los Angeles, CA 90024-1555 \\ vsv@math.ucla.edu \\ Herbert Clemens \\ University of Utah \\ Salt Lake City, UT 84112 \\ clemens@math.utah.edu \\ F. Michael Christ \\ University of California \\ Los Angeles, CA 90024-1555 \\ christ@math.ucla.edu \\ ThOMAs ENRIGHT \\ University of California, San Diego \\ La Jolla, CA 92093 \\ tenright@ucsd.edu
}

\author{
Nicholas ERCOLANI \\ University of Arizona \\ Tucson, AZ 85721 \\ ercolani@math.arizona.edu \\ R. FINN \\ Stanford University \\ Stanford, CA 94305 \\ finn@gauss.stanford.edu \\ VAUGHAN F. R. JONES \\ University of California \\ Berkeley, CA 94720 \\ vfr@math.berkeley.edu \\ Steven KerckhofF \\ Stanford University \\ Stanford, CA 94305 \\ spk@gauss.stanford.edu
}

C. C. Moore

University of California

Berkeley, CA 94720

MaRTin SCHARLEMANN

University of California

Santa Barbara, CA 93106

mgscharl@henri.ucsb.edu

HAROLD STARK

University of California, San Diego

La Jolla, CA 92093

\author{
UNIVERSITY OF ARIZONA \\ UNIVERSITY OF BRITISH COLUMBIA \\ CALIFORNIA INSTITUTE OF TECHNOLOGY \\ UNIVERSITY OF CALIFORNIA \\ MONTANA STATE UNIVERSITY \\ UNIVERSITY OF NEVADA, RENO \\ NEW MEXICO STATE UNIVERSITY \\ OREGON STATE UNIVERSITY
}

\section{SUPPORTING INSTITUTIONS}

UNIVERSITY OF OREGON

UNIVERSITY OF SOUTHERN CALIFORNIA

STANFORD UNIVERSITY

UNIVERSITY OF HAWAII

UNIVERSITY OF TOKYO

UNIVERSITY OF UTAH

WASHINGTON STATE UNIVERSITY

UNIVERSITY OF WASHINGTON 


\section{PACIFIC JOURNAL OF MATHEMATICS}

Volume $156 \quad$ No. $1 \quad$ November 1992

Enveloping algebras of Lie groups with discrete series

Nguyen HuU AnH and VuOng Manh Son

Asymptotic behavior of eigenvalues for a class of pseudodifferential operators on $\mathbf{R}^{n}$

JUNICHI ARAMAKI

A hybrid of theorems of Vinogradov and Piatetski-Shapiro

ANTAL BALOG and JOHN BENJAMIN FRIEDLANDER

Chaos in terms of the map $x \rightarrow \omega(x, f)$

ANDREW MiCHAEL BRUCKNER and JACK GARY CEDER

Local real analytic boundary regularity of an integral solution operator 97 of the $\bar{\partial}$-equation on convex domains

ZHENHUA CHEN

On some properties of exhaustion maps between bounded domains

Chi Keung Cheung

A generalization of maximal functions on compact semisimple Lie groups

HENDRA GUNAWAN

Stability of nonsingular group orbits

CLARK DEAN HORTON

Bordism and regular homotopy of low-dimensional immersions

JOHN FORBES HuGHES

On six-connected finite $H$-spaces

JAMES PEICHENG LiN and Frank WiLliams 